九州大学学術情報リポジトリ

Kyushu University Institutional Repository

Enumeration of the Heteroptera in Mt. Hikosan, Western Japan with Their Hosts and Preys I

Takeno, Koichi

https://doi.org/10.5109/2623

出版情報: ESAKIA. 38，pp. 29-53，1998-03-31. Entomological Laboratory，Faculty of Agriculture， Kyushu University

バージョン :

権利関係 : 


\title{
Enumeration of the Heteroptera in Mt. Hikosan, Western Japan with Their Hosts and Preys I*
}

\author{
Koichi TAKENO \\ Hikosan Biological Laboratory, Faculty of Agriculture, \\ Kyushu University, Hikosan, 824-0721 Japan
}

\begin{abstract}
The bugs of the Heteroptera in Mt. Hikosan, Japan, are enumerated together with their host plants and preys confirmd by the author himself. First part treats 11 families including 83 species in 55 genera.
\end{abstract}

Key words: Heteroptera, list, Mt. Hikosan, hosts, preys.

The present list includes the collection of Heteroptera, which were collected in Mt. Hikosan, and preserved in the Hikosan Biological Laboratory, Kyushu University, together with the records by the previous authors. It also includes new records of hosts and preys. In the lists, double asterisks show the host plants or preys, which were newly recognized and recorded here, and triple asterisks show new records from Mt. Hikosan.

Before going further the author wishes to express his sincere thanks to Prof. Emeritus K. Morimoto, Prof. J. Yukawa, Assoc. Prof. 0. Tadauchi and Assist. Prof. S. Kamitani of Entomological Laboratory, Faculty of Agriculture, Kyushu University, and Assoc. Prof. M. T. Chûjô of Hikosan Biological Laboratory, Faculty of Agriculoture, Kyushu University for their kind guidance in various ways. Especially much thanks are due to former Prof. S. Miyamoto of Kyushu University for correction of manuscript and for examination of every species determined by the author.

$\begin{array}{ll}\text { I. Enicocepharidae } & \text { Jap. name: Kubinagakamemushi-ka } \\ \text { II. Nepidae } & \text { Jap. name: Taikouchi-ka } \\ \text { III. Ochteridae } & \text { Jap. name: Memizumushi-ka } \\ \text { IV. Notonectidae } & \text { Jap. name: Matsumomushi-ka } \\ \text { V. Saldidae } & \text { Jap. name: Mizugiwakamemushi-ka } \\ \text { VI. Miridae } & \text { Jap. name: Mekurakamemushi-ka } \\ \text { VII. Nabidae } & \text { Jap. name: Makibasashigame-ka } \\ \text { VIII. Anthocoridae } & \text { Jap. name: Hanakamemushi-ka } \\ \text { IX. Tingidae } & \text { Jap. name: Gunbaimushi-ka }\end{array}$

* Contribution from the Hikosan Biological Laboratory, Faculty of Agriculture, Kyushu University, Hikosan (Ser. 4, No. 18). 


\author{
X. Reduviidae Jap. name: Sashigame-ka \\ XI. Aradidae Jap. name: Hiratakamemushi-ka
}

\title{
I. ENICOCEPHALIDAE
}

\section{Enicocephalinae}

\section{Hoplitocoris (Pseudenicocephalus) lewisi (Distant, 1883)}

Jap. name: Hime-kubinaga-kamemushi

Spec. exam.: 1 ex., 2. vii. 1070, K. Takeno leg.; 61 exs., (A), 17:50-18:30 P. M. swarming, 4. vii. 1980, K. Takeno leg.

Gen. distr.: Hokkaido, Honshu and Kyushu; Siberia and China.

Prey: Unknown.

\section{Stenopirates japonicus (Esaki, 1935)}

Jap. name: Kuro-kubi-naga-kamemushi

Spec. exam.: 1 ex., 25. vii. 1966, A. Taketani leg.

Gen.distr.: Honshu and Kyushu.

Prey: Unknown.

\section{NEPIDAE}

\section{Ranatrinae}

\section{Ranatra chinensis Mayer, 1865}

Jap. name: Mizu-kamakiri

Spec. exam.: 1 ex., 10. vii. 1948, A. Habu leg.

Gen. distr.: Hokkaido, Honshu, Shikoku, Kyushu and Okinawa Is.; E. Siberia, Russia (Far East), China, Korea, Formosa and Burma.

Prey: Unknown.

\section{OCHTERIDAE}

\section{Ochterus marginatus Latreille, $1807^{* * *}$}

Jap. name: Me-mizumushi

Spec. exam.: 3 exs., 1 nymph, 22. v. 1979, K. Takeno leg.; 28 exs., 24. v. 1979, K. Takeno leg.; 19 exs., 25. v. 1979, K. Takeno leg.

Gen. distr.: Honshu, Shikoku, Kyushu and Iriomote Is.; China, S. Europe, India and N. Australia. 
Host?: Unknown.

\section{NOTONECTIDAE}

\section{Anisopinae}

5. Anisops ogasawarensis Matsumura, 1915 Jap. name: Ko-matsumomushi

Spec. exam.: 1 ex., (A), light trap, 22. vii. 2997, K. Takeno leg.

Gen. distr.: Honshu, Shikoku and Kyushu; China, Korea and Formosa.

Prey: Unknown.

\section{SALDIDAE}

\section{Saldinae}

\section{Saldula saltatoria (Linnaeus, 1758)}

Jap. name: Mizugiwa-kamemushi

Spec. exam.: 1 ex., 2. v. 1937, K. Takeno leg.; 1 ex., 22. vi. 1966, K. Takeno leg.; 1 ex., 12. iv. 1969, K. Kanmiya leg.; 1 ex., 18. v. 1972, K. Takeno leg.; 10 exs., (AM), (sweeping), 22. v. 1979, K. Takeno leg.; 13 exs., 24. v. 1979, K. Takeno leg.; 9 exs., 25. v. 1979, K. Takeno leg.

Gen. distr.: Hokkaido, Honshu, Shikoku, Kyushu and Tsushima Is.; Holoarctic Region and Formosa.

Host?: [Homono-ka: Graminae] Suzumenoteppou: Alopeculus aequalis Sobol.**, Suzu-menokatabira: Poa annua Linnaeus**.

\section{MIRIDAE}

\section{Bryocorinae}

\section{Monalocoris filicis (Linnaeus, 1758)}

Jap. name: Zu-aka-shida-mekuragame

Spec. exam.: 2 exs., 7. xi. 1938, K. Yasumatsu leg.; 1 ex., 21. vii. 1968, K. Kanmiya leg.; 1 ex., 1. viii. 1968, K. Kanmiya leg.; 1 ex., 25. viii. 1968, K. Kanmiya leg.; 7 exs., (G), 13. ix. 1972, K. Takeno leg.; 8 exs., (A), 25. i. 1978, K. Takeno leg.; 8 exs., (A), 25. iii. 1980, K. Takeno leg.; 1 ex., (A), light trap, 15. ix. 1980, K. Takeno leg.; 4 exs., (V), 17. ix. 1980, K. Takeno leg.; 2 exs., (A), beeting, 30. i. 1981, K. Takeno leg. 
Gen. distr.: Hokkaido, Honshu, Shikoku, Kyushu and Ryukyus; Russia (Far East) and Formosa.

Host: [Uraboshi-ka: Polypodiaceae] Shikechishida: Athyrium decurrentialatum Makino leg.; Oohimewarabi: Athyrium okuboanus Makino, Itachishida: Dryopteris variu 0. Kuntze, Kumawarabi: Dryopteris lacera 0. Kuntze**, Inodemodoki: Polystichum tagawanum Kurata** (Tsutsui), Yabusotetsu: Polystichum Fortunei Nakai**, Jyuumonjishida: Polystichum tripteron Presl, Yamainuwarabi: Athyrium Vidulii Nakai**.

\section{Deraeocorinae}

\section{Termatophylum hikosanum Miyamoto, 1965}

Jap. name: Hikosan-tengu-mekuragame

Spec. exam.: 1 ex., 5. ix. 1964, K. Takeno leg.; 1 ex., 2 1. v. 1975, K. Takeno leg.; 1 ex. light trap, (A), 23. vii. 1997, K. Takeno leg.; 1 ex. light trap, (A), 31. vii. 1997, K. Takeno leg.

Gen. distr.: Shikoku and Kyushu.

Host: [Toudaigusa-ka: Euphorbiacea] Akamegashiwa: Mallotus japonicus Muell. Arg. [Kabanoki-ka: Betulaceae] Inushide: Curpinus Tschonoskii Maxim., Akashide: Curpinus laxiflora Blume, Kumashide: Curpinus curpinoides Makino, Sawashiba: Curpinus erosa Blue; Prey: [Azamiuma-ka Thripidae] Azamiuma: Thrips spp.

\section{Mirinae}

9. Adelphocoris albonotatus (Jakovlev, 188 1)

Jap. name: Yotsumon-mekuragame

Spec. exam.: 1 ex., 7. vi. 1937, K. Yasumatsu leg. (through by Yasunaga, 1990).

Gen. distr.: Hokkaido, Honshu, Shikoku, Kyushu and Goto Iss.; Russia (Far East), China, Korea and India.

Host: [Mame-ka: Reguminosae] Yamahagi: Lespedeza bicolor Trucz. var. japonica Nakai.

\section{Adelphocoris demissus Horvath, 1905}

Jap. name: Usumon-mekuragame

Spec. exam.: 1 ex., 19. viii. 1938, K. Yasumatsu leg.; 1 ex., 8. vii. 1939, K. Yasumatsu leg.; 1 ex., (M-O), 17. ix. 1974, K. Takeno leg.; 1 ex., (A), light trap, 4. vii. 1975, K. Takeno leg.; 1 ex., (0), 10. ix. 1979, K. Takeno leg.

Gen. distr.: Hokkaido, Hosnhu, Shikoku and Kyushu.

Host: [Mame-ka: Reguminosae] Yamahagi: Lespedeza bicolor Turcz. var. juponicu Nakai. 


\section{Adelphocoris piceosetosus Kulik, 1965}

Jap. name: Usuka-mekuragame

Spec. exam.: None (through by Yasunaga, 1990).

Gen. distr.: Hokkaido, Honshu, Shikoku and Kyushu; Kuril Iss., Russia (Far East) and Korea.

Host: [Mame-ka: Reguminosae] Yamahagi: Lespedeza bicolor Trucz. var. japonica Nakai.

\section{Adelphocoris lineolatus (Goeze, 1778)}

Jap. nae: Ususuji-mekuragame

Spec. exam.: 1 ex., 10. vi. 1937, K. Yasumatsu leg.; 5 exs., 8. vii. 1939, K. Yasumatsu leg.; 3 exs., (G), 22. ix. 1972, K. Takeno leg.

Gen. distr.: Hokkaido, Honshu, Shikoku and Kyushu; China, Korea and Holoarctic Region.

Host: [Mame-ka: Leguminosae] Murasakiumagoyashi: Medicago sutivu Linnaeus; [Tade-ka: Polygonaceae] Mizosoba: Polygonum Thunhergii Sieb. et Zucc., [Homono-ka: Graineae] Enokorogusa: Setaria viridis Beauv.

\section{Adelphocoris suturalis (Jakovlev, 1882)}

Jap. name: Nakaguro-mekuragame

Spec. exam.: 1 ex., 6. vi. 1937, K. Yasumatsu leg.; 1 ex., 3. ix. 1937, K. Yasumatsu leg.; 1 ex., 6. v. 1939, K. Yasumatsu leg; 1 ex., 8. vii. 1939, K. Yasumatsu leg.; 2 exs., 27. vi. 1968, K. Kanmiya leg.; 1 ex., (A), light trap, 2. viii. 1975, K. Takeno leg.; 1 ex., (A), light trap, 13. ix. 1975, K. Takeno leg.; 1 ex., (0), 10. ix, 1979, K. Takeno leg.; 1 ex., 1 vi. 1995, K. Takeno leg.

Gen. distr.: Hokkaido, Honshu, Shikoku and Kyushu; Kuril Iss., E. Siberia, Russia (Far East), Sakhalin, China and Korea.

Host: [Kiku-ka: Compositae] Yomogi: Artemisiu vulgaris L. var. indica Maxim., Mibuyomogi: Artemisia muritimu Linnaeus (through Plants of the World), Shunngiku: Chrysanthemum coronurium L. var. sputiosum Bailey, Mokushunngiku: Chrysuntheum frutescens Linnaeus, [Mame-ka: Leguminosae] Azuki: Phuseolus angularis Wight, Daizu: Glycine Max Merrill, Yamahagi: Lespedezu bicolor Trucz. var. juponicu Nakai, Tsuruinngenn: (local name), [Kayatsurigusa-ka: Cyperaceae] Kayatsurigusa: Cyperus microriu Steud., [Homono-ka: Gramineae spp.] Ine-ka: Oryza sutivu Linnaeus.

\section{Adelphocoris triannulatus (Stal, 1858)}

Jap. name: Buchihige-kuro-mekuragame

Spec. exam.: 1 ex., 10. vi. 1937, K. Yasumatsu leg.; 2 exs., 6. vii. 1939, K. Yasumatsu leg.; 2 exs., 4. vii. 1950, A. Habu leg.; 1 ex., (A), 2. ix. 1974, K. Takeno leg.; 1 ex., (V), 5. xi. 1975, K. Takeno leg.; 1 ex., (A), 24, vii., 1980, K. Takeno leg.; 
1 ex., (A), 4. ix. 1980, K. Takeno leg.; 1 ex., (V), 17. ix. 1980, K. Takeno leg.; 1 ex., (A), 8. v. 1983, K. Takeno leg.; 2 exs., (U), 17. ix. 1983, K. Takeno leg.; 1 ex., (A), 11. x. 1983, K. Takeno leg.; 3 exs., (U), 18. x. 1983, K. Takeno leg.

Gen. distr.: Hokkaido, Honshu, Shikoku, Kyushu and Goto Iss.; Kuril Iss., Russia (Far East), Sakhalin, China and Korea.

Host: [Kiku-ka: Compositae] Yomogi: Artemisia vulgaris Linnaeus, [Mame-ka: Leguminosae] Ingenmame: Dolichos lablab Linnaeus, Yamahagi: Lespedeza bicolor Trucz. var. japonica Nakai, Komatsunagi: Indigoferadecora Lindl., [Yanagi-ka: Salicaceae] Willow: Salix spp., [Yuri-ka: Liliaceae] Nira: Alium odorum Linnaeus, [Hoono-ka: Gramineae] Ine: Oriza sativa Linnaeus, Nezumimugi: Lolium multiflorum Lam.(through Plants of the World).

\section{Arbolygus rubripes (Jakovlev, 1876)}

Jap. name: Ashiaka-kuro-mekuragame

Spec. exam.: 1 ex., 9. vi. 1937, K. Yasumatsu leg.; 1 ex., 21. viii. 1956, T. Esaki leg.; 1 ex., (A), 28. vii. 1983, K. Takeno leg.

Gen.distr.: Hokkaido, Honshu, Kyushu and Yakushima Is.; Kuril Iss. and Russia (Far East).

Host: [Mame-ka: Leguminosae] Ingenmame: Dolichos lablab Linnaeus, Sasage: Vigna catiang Endl. var. sinensis King, Azuki: Phaseolus angularis Wight., Daizu: Glucine max Merrill, [Tade-ka: Polygonaceae] Mizosoba: Polygonum Z’hunbergii Sieb. et Zucc.**, [Homono-ka: Gramineae] Suzutake: Pseudosasa purpurascens Makino, Chishimazasa: local name), Chishimanezasa (local name), [Yanagi-ka: Salicaceae] Yanagi: Salix spp.

16. Charagochilus gyllenhalii (Fallen, 1807)

Jap. name: Hime-sedaka-mekuragame

Spec. exam.: 1 ex., (V), 11. x. 1983, K. Takeno leg.; lex., (A), 18. v. 1988, K. Takeno leg.

Gen. distr.: Hokkaido, Honshu, Shikoku and Kyushu; Palaearctic Region.

Host: [Mame-ka: Reguminosae] Yabutsuruazuki: Phaseolus trilobatus Schreb**.

17. Creontiades eurytus Yasunaga, 1988

Jap. name: Nise-oochairo-mekuragame

Spec. exam.: 1 ex., (A), light trap, 2. viii. 1975, K. Takeno leg. Gen. distr.: Honshu and Kyushu.

Host: Unknown.

18. Creontiades pallidifer (Walker, 1873)

Jap. name: Aka-hoshi-mekuragame 
Spec. exam.: 1 ex., 19. viii. 1938, K. Yasumatsu leg.; 1 ex., 7. vii. 1950, A. Habu leg.

Gen. distr.: Honshu, Shikoku, Kyushu, Tokara Iss. and Bonin Iss.; Formosa, S.-E. Asia and Micronesia.

Host: [Mame-ka: Reguminosae] Daizu: Glycine Max Merrill, Yahazusou: Microlespedeza striata Makino, Yamahagi: Lespedeza bicolor Trucz. var. japonica Nakai, [Homono-ka: Graineae] Ine: Oryza sativa Linnaeus.

\section{Creontiades tricolor (Scott, 1880)}

Jap. name: Oochairo-mekuragame

Spec. exam.: 1 ex., 26. vii. 195 1, A. Habu leg.; 1 ex., 7. ix. 1970, K. Takeno leg.; 1 ex., (A-Z), 13. vi. 1974, K. Takeno leg.; 1 ex., 22. vi. 1974, K. Ohara leg.; 1 ex., 4. vii. 1975, K. Takeno leg.; 1 ex., (D), 11. ix. 1975, K. Takeno leg.; 1 ex., (A), 14. ix. 1975, K. Takeno leg.; 1 ex., (A), 9. v. 1983, K. Takeno leg.

Gen. distr.: Hokkaido, Honshu, Shikoku, Kyushu and Tsushima Is.; Russia (Far East).

Host: [Buna-ka: Fagaceae] Mizunara: Quercus crispula Blume, Kunugi: Quercus acutissima Carruth, [Yanagi-ka: Salicaceae] Yanagi: Salix spp.

\section{Eolygus rubrolineatus (Matsumura, 19 13)}

Jap. name: Akasuji-higebuto-mekuragame

Spec. exam.: 2 exs., 9. vi. 1937, K. Yasumatsu leg.; 1 ex., 2. vi. 1939, K. Yasumatsu leg.; 5 exs., 5. vii. 1939, K. Yasumatsu leg.; 1 ex., 29. vii. 1963, S. Kimoto leg.; 1 ex., 13. iv. 1964, S. Kimoto leg.; 1 ex., 15. vi. 1964, S. Kimoto leg.; 1 ex., 4. vii. 1969, M. T. Chûjô leg.; 12 exs., (A), 29. vi. 1974, K. Kanmiya leg.; 13 exs., 12. vii. 1974, K. Takeno leg.; 1 ex., (D-E), 6. x. 1975, K. Takeno leg.; 1 ex., (V), 7. vii. 1976, K. Takeno leg.; 1 ex., (V), 2. v. 1978, K. Takeno leg.; 1 ex., (A), 10. viii. 1978, K. Takeno leg.; 1 ex., (A), 1. vii. 1980, K. Takeno leg.

Gen. distr.: Hokkaido, Honshu, Shikoku and Kyushu; Russia (Far East).

Host: [Kiku-ka: Compositae] Yakushisou: Lacutuca denticulata Maxim.**, Onitabirako: Crepis japonica Benth. **, Himejoon: Erigeron annuus Linnaeus**, Nigana: Lactuca dentata Makino, [Karakasabana-ka: Umbelliferae] Mitsuba: Cryptotaenia canadensis Dc.**; [Shida-rui: Eufilicales spp.].

\section{Eurystylus coelestialium (Kirkaldy, 1902)***}

Jap. name: Mengata-mekuragame

Spec. exam.: 1 ex., 1. x. 1964, K. Takeno leg.; 2 exs., (X), 9. vi. 1974, K. Takeno leg.; 2 exs., 26. vi. 1974, K. Takeno leg.; 7 exs., (M-O), 17. ix. 1974, K. Takeno leg.; 2 exs., (A), 17. vi. 1976, K. Takeno leg. 
Gen. distr.: Hokkaido, Honshu, Shikoku and Kyushu; Kuril Iss., Russia (Far East), China and Korea.

Host: [Hiiragi-ka: Oleaceae] Nezumimochi: Ligustrumjuponicum Thunb., [Utsugi-ka: Araliaceae] Taranoki: Aralia elata Seem.**, Udo: Aralia cordata Thunb., [Mitsubautsugika: Staphyleaceae] Gonzui: Euscuphis juponicu Pax.**, [Mame-ka: Leguminosae] Yamahagi: Lespedeza bicolor Trucz. var. juponicu Nakai, [Yukinoshita-ka: Saxifragaceae] Noriutsugi: Hydrangea paniculata Sieb., [Kuwa-ka: Moraceae] Yamaguwa: Morus bombycis Koidz. (Ooi).

\section{Gigantomiris jupiter Miyamoto et Yasunaga, 1988}

Jap. name: Akasuji-oo-mekuragame

Spec. exam.: 2 exs., 18. vi. 1937, K. Yasumatsu leg.

Gen. distr.: Honshu, Shikoku and Kyushu; Korea and Russia (Coastal area).

Host: [Suikazura-ka: Caprifoliaceae] Suikazura: Loniceru juponicu Thunb., [Buna-ka: Fagaceae] Mizunara: Quercus crispula Blume, [Kabanoki-ka: Betulaceae] Mizume: Betula grossa Sieb. et Zucc., Onigurumi: Juglans mundshricu Maxim. var. Siebldiana Makino, [Yanagi-ka: Salicaceae] Salix spp.

\section{Lygocoris (Apolygus) hilaris (Horvath, 1905)}

Jap. name: Futamon-aka-mekuragame

Spec. exam.: 1 ex., 3. ix. 1938, K. Yasumatsu leg.; 1 ex., 27. v. 1938, T. Esaki, K. Nomura \& K. Yasumatsu leg.: 1 ex., (A), light trap, 29. v. 1974, K. Ohara leg.; 14 exs., (A), 23. v. 1975, K. Takeno leg.; 2 exs., (A), light trap, 5. vi. 1975, K. Takeno leg.; 1 ex., (A), light trap, 13. vi. 1975, K. Takeno leg.; 2 exs., (A), light trap, 19. vi. 1975, K. Takeno leg.: 2 exs., (A), light trap, 6. viii. 1975, K. Takeno leg.; 1 ex., 13. ix. 1975, K. Takeno leg.; 6 exs., (A), light trap, 14. ix. 1975, K. Takeno leg.; 4 exs., (A), light trap, 24. v. 1978, K. Takeno leg.; 3 exs., 11. ix. 1979, K. Takeno leg.; 1 ex., (A), light trap, 18. ix. 1980, K. Takeno leg.; 1 ex., (A), 30. v. 1984, K. Takeno leg., 1 ex., (A), light trap, 8. vii. 1987, K. Takeno leg.

Gen. distr.: Hokkaido, Honshu, Shikoku and Kyushu; Russia (Far East) and Korea.

Host: [Mame-ka: Leguminosae] Marubahagi: Lespedezu cyrtobotryu Miq., [Ibara-ka: Rosaceae] Ushikoroshi: Pourthiueu villosu Decne**.

\section{Lygocoris (Apolygus) lucorum (Meyer-Dur, 1843)}

Jap. name: Ko-ao-mekuragame

Spec. exam.: 1 ex., 8. vii. 1939, K. Yasumatsu leg.; 1 ex., (A), 14. ix. 1975, K. Takeno leg.; 1 ex., (A), 12. ix. 1978, K. Takeno leg.; 2 exs., (0), 10. ix. 1979, K. Takeno leg.; 2 exs., (V), sweeping in paddy field, 3. x. 1980, K. Takeno leg.; 1 ex., (A), light trap, 8. vii. 1987, K. Takeno leg. 
Gen. distr.: Hokkaido, Honshu, Shikoku and Kyushu; Russia (Far East), China, Korea, Europe and N. America.

Host: [Kiku-ka: Compositae] Kiku: Chrysanthemum morifolium Ramat. var. sinensis Makino, Yomogi: Artemisia vulgaris L. var. indica Maxim., [Uri-ka: Cucurbitaceae] Suika: Citrullus vulgaris L., Kyuuri: Cucumis sativus L., [Nasu-ka:] Nasu: Solanum melongena L. var. esculentu Nees, [Tsubaki-ka: Theaceae] Cha: Thea sinensis L., [Budou-ka: Vitaceae] Budou: Vitis vinifera L.

\section{Lygocoris (Apolygus) nigritulus (Linnavuori, 1961)}

Jap. name: Kuroba-mekuragame

Spec. exam.: 1 ex., (A), light trap, 30. vii. 1968, K. Kanmiya leg.; 1 ex., 1. viii. 1968, K. Takeno leg.; 1 ex., 16. vi. 1969, M. T. Chûjô leg.; 2 exs., 17. v. 1971, K. Takeno leg.; 1 ex., 28. vi. 1971, K. Takeno leg.; 3 exs., (A-D), 24. iv. 1974, K. Takeno leg.; 1 ex., (M-O), 17. ix. 1974, K. Takeno leg.; 1 ex., 26. v. 1974, K. Ohara leg.; 1 ex., (A-M), 27. v. 1975, K. Takeno leg.; 1 ex., (A), 22. vii. 1977, K. Takeno leg.; 3 exs., (D-E), 26. ix. 1977, K. Takeno leg.; 1 ex., (A), light trap, 12. ix. 1978, K. Takeno leg.; 3 ex., (C), 4. x. 1978, K. Takeno leg.; 1 ex., (A), 23. iv. 198 1, K. Takeno leg.; 1 ex., (V), 11. x. 1983, K. Takeno leg.; 1 ex., (F), 4. vi. 1985, K. Takeno leg.

Gen. distr.: Hokkaido, Honshu, Shikoku and Kyushu; China and Korea.

Host: [Kuchibirubana-ka: Labiatae] Akinotaurasou: Salvia chinensis Benth.**, [Kaede-ka: Aceraceae] Irohakaede: Acer palmatum Thunb.**, [Irakusa-ka: Urticaceae] Koakaso: Boehmeria spicata Thunb.

26. Lygocoris (Apolygus) pallens Yasunaga, 1991

Jap. name: Koakaso-mekuragame

Spec. exam.: 1 ex., 10. vii. 1939, K. Yasumatsu leg.; 1 ex., 4. vii. 1950, A. Habu leg.; 1 ex., (A), 27. viii. 1973, K. Takeno leg.; 2 exs., (A), light trap, 9. vi. 1987, K. Takeno leg.

Gen. distr.: Honshu, Shikoku and Kyushu.

Host: [Irakusa-ka: Urticaceae] Koakaso: Boehmeriu spicata Thunb.

\section{Lygocoris (Apolygus) subhilaris Yasunaga, 1992}

Jap. name: Nise-futamon-aka-mekuragame

Spec. exam.: 1 ex., 5. viii. 1987 (L.T.), T. Yasunaga leg; 1 ex., 3-4. viii. 1988 (L.T.), T. Yasunaga leg. (through Yasunaga, 1992).

Gen. distr.: Honshu and Kyushu.

Host: Unknown.

28. Lygocoris (Apolygus) subpulchellus Kerzhner, 1987

Jap. name: Tsumaguro-hagi-mekuragame 
Spec. exam.: 1 ex., elev. 650m, 6. vi. 1938, K. Yasumatsu leg.; 1 ex., 19. viii. 1938, K. Yasumatsu leg.; 1 ex., 12. vii. 1939, K. Yasumatsu leg.; 2 exs., 28. vii. 1939, K. Yasumatsu leg.; 1 ex., 22. viii. 1948, T. Esaki leg.; 1 ex., 29. vii. 1963, S. Kimoto leg.; 2 exs., 3. ix. 1964, S. Kimoto leg.; 3 exs., 5. ix. 1964, S. Kimoto leg.; 2 exs., 7. ix. 1964, S. Kimoto leg.; 1 ex., 10. ix. 1968, K. Kanmiya leg.; 1 ex., 13. ix. 1969, K. Kanmiya leg.; 1 ex., 27. vii. 1970, K. Takeno leg.; 3 exs., (A), light trap, 11. viii. 1970, M. T. Chûjô leg.; 1 ex., 20. viii. 1970, K. Takeno leg.; 1 ex., 21. viii. 1970, M. T. Chûjô leg.: 5 exs., (A), light trap, 3. ix. 1970, K. Takeno leg.; 4 exs., (A), light trap, 7. ix. 1970, K. Takeno leg.; 16 exs., (A), light trap, 27. viii. 1973, K. Takeno leg.; 1 ex., (A), light trap, 5. vi. 1975, K. Takeno leg.; 7 exs., (A), light trap, 13. vi. 1975, K. Takeno leg.; 15 exs., (A), light trap, 19. vi. 1975, K. Takeno leg.; 3 exs., (A), light trap, 3. vii. 1975, K. Takeno leg.; 1 ex., (A), 7. vii. 1975, K. Takeno leg.; 1 ex., (A), light trap, 25. vii. 1975, K. Takeno leg.; 1 ex., (A), 4. viii. 1975, K. Takeno leg.; 7 exs., (A), light trap, 5. viii. 1975, K. Takeno leg.; 1 ex., (A), 6. viii. 1975, K. Takeno leg.; 23 exs., (A), light trap, 13. ix. 1975, K. Takeno leg.; 17 exs., (A), light trap, 14. ix. 1975, K. Takeno leg.; 1 ex., (A), 1. viii. 1977, K. Takeno leg.; 1 ex., (A), light trap, 29. viii. 1977, K. Takeno leg.; 1 ex., 28. viii. 1978, K. Takeno leg.; 10 exs., (A), light trap, 31. viii. 1978, K. Takeno leg., 11 exs., (A), 12. ix. 1978, K. Takeno leg.; 1 ex., (A), 29. vii. 1979, K. Takeno leg.; 1 ex., (A), 7. viii. 1979, K. Takeno leg.; 1 ex., (A), light trap, 15. ix. 1979, K. Takeno leg.

Gen. distr.: Honshu, Shikoku, Kyushu and Tsushima Is.; Russia (Far East).

Host: [Kiku-ka: Compositae] Himejoon: Erigeron annuus Linnaeus, [Mame-ka: Leguminosae] Yamahagi: Lespedeza bicolor Trucz. var. japonica Nakai, Miyaginohagi: Lespedeza Thunbergii Nakai**, [Buna-ka: Fagaceae] Tsuburajii: Shiia cuspidata Makino, [Mikan-ka: Rutaceae]. Gen. et sp.

\section{Lygocoris (Neolygus) honshuensis (Linnavuori, 196 1)}

Jap. name: Futamon-usuki-mekuragame

Spec. exam.: 1 ex., 29. vii. 1963, S. Kimoto leg.; 1 ex., (A), light trap, 22. vi. 1974, K. Ohara leg.; 1 ex., (A), light trap, 5. vi. 1975, K. Takeno leg.; 1 ex., (A), 19. vi. 1975, K. Takeno leg.; 1 ex., (A), 14. ix. 1975, K. Takeno leg.; 1 ex., (A), 30. viii. 1983, K. Takeno leg.

Gen. distr.: Hokkaido, Honshu and Kyushu; Kuril Iss. and Sakhalin.

Host: [Hiiragi-ka: Oleaceae] Hashidoi: Syringa amurensis Rupr. var. japonica Franch. et Sav., [Yukinoshita-ka: Saxifragfaceae] Noriutsugi: Hydrangea paniculata Sieb.

\section{Lygocoris (Neolygus) kyushuensis Yasunaga, 1991}

Jap. name: Minami-midori-mekuragame

Spec. exam.: 2 exs., (B-C), 25. vi. 1948, K. Yasumatsu leg.; 3 exs., 1. vii. 1950, A. Habu leg.; 10 exs., 6. vi. 1064, S. Kimoto leg.; 1 ex., (A), light trap, 13. vi. 1966, K. 
Takeno leg.; 1 ex., (A), light trap, 13. vi. 1975, K. Takeno leg.; 4 exs., (A), light trap, 19. vi. 1975, K. Takeno leg.

Gen. distr.: Kyushu, Yakushima Is., Amami-Oshima Is. and Okinawa Is.

Host: [Buna-ka: Fagaceae] Tsuburajii: Shiia cuspidata Makino.

\section{Lygocoris (Neolygus) obesus Yasunaga, 1991}

Spec. exam.: 1 ex., 9. vii. 1987 (L.T.), K. Takeno leg.; 1 ex., 18. viii. 1975 (L.T.), K. Takeno leg.; 1 ex., 23. vii. 1977, (L.T.), K. Takeno leg. (through Yasunaga, 1991). Gen. distr.: Kyushu.

Host: Unknown.

\section{Lygus saundersi Reuter, 1896}

Jap. name: Madara-mekuragame

Spec. exam.: 1 ex., 30. v. 1948, K. Yasumatsu leg.: 3 exs., 1. vii. 1950, A. Habu leg.; 10 exs., 6. vi. 1964, S. Kimoto leg.; 1 ex., (A), light trap, 13. vi. 1966, K. Takeno leg.; 1 ex., 12. iv. 1967, K. Takeno leg.; 1 ex., 20. vi. 1969, K. Takeno leg.; 1 ex., (A), light trap, 13. vi. 1975, K. Takeno leg.

Gen. distr.: Hokkaido, Honshu, Shikoku, Kyushu and Tsushima Is.; Kuril Iss., Russia (Far East), Sakhalin, China and Korea.

Host: [Kayatsurigusa-ka: Cyperaceae] Aibasou: Scirpuscyperinus Kunth var. Wichurai Makino, Kuroaburagaya: Scirpus orientalis Ohwi (Ooi), [Homono-ka: Gramineae] Suzu-menoteppou: Alopeculus aequalis Sobol., Komugi: Triticum uestivum Linnaeus.

\section{Mermitelocerus annulipes Reuter, 1908***}

Jap. name: Shima-ao-mekuragame

Spec.exam.: 1 ex., beeting in paddy field, (A), 2. vi. 1981, K. Takeno leg.

Gen. distr.: Hokkaido, Honshu, Shikoku and Kyushu; Kuril Iss., Russia (Far East), Sakhalin and China.

Host: [Kiku-ka: Compositae] Azami spp.: Çbirsium sp. (Ooi), [Mame-ka: Leguminosae] Nodafuji: Wistaria floribunda DC., Yamafuji: Wisturiu bruchybotrys Sieb. et Zucc., [Jyuujibana-ka: Cruciferae] Konronsou: Dentaria macrophylla Bunge var. dasyloba Makino, Oobadakefuji: (local name); Prey: [Madaraga-ka: Zygaenidae (Ins., Lepidoptera)] Kisujihosomadara: Artonu gracilis Walker (nymph).

\section{O nomaus lautus (Uhler, 1896)}

Jap. name: Aka-ashi-mekuragame

Spec. exam.: 1 ex., 15. vii. 1943, S. Ito leg.; 2 exs., 29. vii. 1963, S. Kimoto leg.; 5 esx., 7. ix. 1964, K. Takeno leg.; 10 exs., 7. ix. 1964, S. Kimoto leg.; 1 ex., 15. ix. 
1964, S. Kimoto leg.; 1 ex., 15. ix. 1964, K. Takeno leg.; 1 ex., 4. viii. 1966, S. Kimoto leg.; 9 exs., 20. vii., 1966, S. Kimoto leg.; 1 ex., 1 1. ix. 1967, S. Kimoto leg.; 4 exs., 24. vii. 1968, K. Takeno leg.; 1 ex., 25. vii. 1968, K. Kanmiya leg.; 1 ex., 8. ix. 1968, K. Kanmiya leg.; 1 ex., 4. vii. 1969, M. T. Chûjô leg.; 1 ex., 11. vii. 1969, K. Kanmiya leg.; 1 ex., 28. vii. 1969, K. Kanmiya leg.; 1 ex., 6. viii. 1969, K. Kanmiya leg.; 1 ex., 4. vii. 1970, M. T. Chûjô leg.; 3 exs., 7. vii. 1971, M. T. Chûjô leg.; 1 ex., (D), 28. viii. 1973, K. Takeno leg.; 1 ex., 3. vii. 1974, K. Takeno leg.; 1 ex., (A), 23. vii. 1974, K. Takeno leg.; 1 ex., (A), 17. ix. 1974, K. Takeno leg.; 2 exs., 18. ix. 1974, K. Takeno leg.; 3 exs., (D), 25. viii. 1975, K. Takeno leg.; 5 exs., 2. ix. 1975, K. Takeno leg.; 3 exs., (A), 22. vii. 1977, K. Takeno leg.; 3 exs., (D), 13. ix. 1977, K. Takeno leg.; 3 exs., (D-E), 26. ix. 1977, K. Takeno leg.; 1 ex., (D), 22. ix. 1978, K. Takeno leg.

Gen. distr.: Hokkaido, Honshu, Shikoku and Kyushu.

Host: [Kiku-ka: Compositae] Noazami: Cirsium juponicum DC.**, Otakarakou: Ligularia sibirica Cadss**, Yomogi: Artemisia vulgar-is L. var. indica Maxim.**, [Oobako-ka: Plantaginaceae] Oobako: Plantago major L. var. asiatica Decne.**, [Kuchibirubana-ka: Labiatae] Akinotamurasou: Salvia chinensis Benth.**, [Murasaki-ka: Borraginaceae] Hi-reharisou: Symphytum officinale L.**, [Akabana-ka: Oenotheraceae] Mizutamasou: Circaea quadrisulcata Franch. et Sav., [Otogirisou-ka: Guttiferae] Kinshibai: Hypericumpatulum Thunb.**, [Housenka-ka: Balsaminaceae] Tsurifunesou: Impatiens Textori Miq., Housenka: Ipatiens Balsamina L.**, [Yukinoshita-ka: Saxifragaceae] Utsugi: Deutzia crenata Sieb. et Zucc. **, Chidakesashi: Astilbe micropylla Knoll., [Jyuujibana-ka: Cruciferae] Konronsou: Dentaria macrophylla Bunge var. dasyloba Makino**, [Kitsunenobotan-ka: Ranunculaceae] Umanoashigata: Ranunkulus acris L. var. juponicus Maxim.**, [Hiyu-ka: Amaranthaceae] Inokozuchi: Achyrunthes japonica Nakai**, [Irakusa-ka: Urticaceae] Koakaso: Boehmeria spicata Thunb.**, Ramii: Boehmeria nivea Gaud. var. candicans Wedd. **, Irakusa: Urtica Thunbergiana Sieb. et Zucc., [Homono-ka: Gramineae] Ine: Oliza sativa Linnaeus.

35. Orthops udonis (Matsumura, 1917)

Jap. name: Monki-makiba-mekuragame

Spec. exam.: 6 exs., vii. 1939, K. Yasumatsu leg.; 1 ex., 8. vii. 1939, K. Yasumatsu leg.; 4 exs., 4. viii. 1950, A. Habu leg.

Gen. distr.: Hokkaido, Honshu and Kyushu; Kuril Iss., Siberia, Russia (Far East), Sakhalin, Mongolia, China and Korea.

Host.: [Ukogi-ka: Araliaceae] Udo: Aralia cordata Thunb., [Karakasabana-ka: Umbelliferae] Miyamashishiudo: Angelica Matsumurae Yabe (Ooi).

36. Phytocoris ohataensis Linnavuori, 1963***

Jap. name: Oo-madara-mekuragame 
Spec. exam.: 21 exs.,(A), light trap, 17. vii. 1975, K. Takeno leg.; 4 exs., (A), light trap, 19. viii. 1977, K. Takeno leg.; 2 exs., (A), light trap, 29. viii. 1977, K. Takeno leg.; 1 ex., (A), 10. viii. 1978, K. Takeno leg.

Gen. distr.: Hokkaido, Honshu, Shikoku and Kyushu; Kuril Iss., Russia (Far East) and Sakhalin.

Host: Unknown.

\section{Stenodema (Brachystira) calcaratum (Fallen, 1807)}

Jap. name: Mugi-mekuragame

Spec. exam.: 1 ex., 4. vii. 1950, A. Habu leg.

Gen. distr.: Hokkaido, Honshu, Shikoku and Kyushu; Palaearctic Region.

Host: [Homono-ka: Gramineae] Suzumenoteppou: Alopeculus prutensis Linnaeus, Ine: Oryza sativa Linnaeus, Mugi-rui: Fusarium spp.

\section{Stenodema rubrinerve Horvath, 1905}

Jap. name: Aka-myaku-mekuragame

Spec. exam.: 1 ex., elev. 650m, 6. vi. 1938, K. Yasumatsu leg.; 1 ex., 19. viii. 1938, K. Yasumatsu leg.; 1 ex., 31. vii. 1948, A. Habu leg.; 1 ex., 21. viii. 1956, H. Kuroko leg.; 1 ex., 4. vi. 1966, S. Kimoto leg.; 1 ex., 20. vii. 1967, K. Takeno leg.; 1 ex., (A), light trap, 27. viii. 1973, K. Takeno leg.; 1 ex., (A), light trap, 28. viii. 1974, K. Takeno leg.; 8 exs., (A), 2. ix. 1974, K. Takeno leg.; 1 ex., (M-O), 17. ix. 1974, K. Takeno leg.; 1 ex., (A-M), 27. v. 1975, K. Takeno leg.; 3 exs., (A), light trap, 6. vi. 1975, K. Takeno leg.; 1 ex., 13. vi. 1975, K. Takeno leg.; 16 exs., (A), light trap, 19. vi. 1975, K. Takeno leg.; 5 exs., (A), 23. vi. 1975, K. Takeno leg.; 1 ex., (A), 13. ix. 1975, K. Takeno leg.

Gen. distr.: Hokkaido, Honshu, Shikoku and Kyushu; Korea.

Host: [Homono-ka: Graineae] Toumorokoshi: Zea mays Linnaeus, Kaya:Miscanthus sinensis Anadedrss., Todashiba: Arundinella hirta Tanaka, Kibi: Panicum miliaceum Linnaeus, Hie: Panicum Crusgalli L. var. frumentaceum Trin., Chikarashiba: Pennisetum japonicum Trin., Kanitsurigusa: Trisetum bifidum Ohwi, Ashi: Phragmites communis Trin., Urahagusa: Hakonechloa macra Makino**, Oomugi: Hordeum vulgare L. var. hexastichon Aschers., Ine: Oryza sativa Linnaeus, Nezumigusa: Lolium multiflorum Lam. (Plants of the World), Mugi-rui: Fusarium spp.

\section{Tinginotum perlatum Linnavuori, 1961***}

Jap. name: Kebuka-mekuragame

Spec. exam.: 1 ex., 26. vii. 1951, A. Habu leg.; 12 exs., 30. ix.-1. x. 1992, K. Takeno leg.; 5 exs., (A), light trap, 1. x. 1992, K. Takeno leg.; 1 ex., 15. vi. 1995, K. Takeno leg.

Gen. distr.: Shikoku and Kyushu. 
Host: Unknown.

40. Taylorilygus pallidulus (Blanchard, 1852)

Jap. name: Usumon-midori-mekuragame

Spec. exam.: 1 ex., (A), sweeping, 2. xi. 1979, K. Takeno leg.

Gen. distr.: Honshu, Shikoku, Kyushu, Tsushima Is., Goto Iss., Nansei Iss. and Bonin Iss.; Cosmopolitan.

Host: [Kiku-ka: Compositae] Yomogi: Artemisia vulgaris Linnaeus, Arechinogiku: Erigeron linifolium Willd.

\section{Phylinae}

\section{1. Hallodapus linnavuori Miyamoto, 1966}

Jap. name: Monshiro-chibi-mekuragame

Spec. exam.: 1 ex., $700 \mathrm{~m}$ in height, 23-25. viii. 1961, S. Miyamoto (L.T.) (through Miyamoto, 1966).

Gen. distr.: Hokkaido, Shikoku and Kyushu; Kuril Iss.

Host: Unknown.

42. Pilophorus miyamotoi Linnavuori, 1961

Jap. name: Matsu-hyoutan-mekuragame

Spec. exam.: 1 ex., 11. vii. 1964, K. Takeno leg.; 1 ex., 4. ix. 1964, K. Takeno leg.

Gen. distr.: Honshu, Kyushu and Tsushima Is.; Russia (Far East).

Host: [Matsu-ka: Pinaceae] Akamatsu: Pinus densiflora Sieb. et Zucc., Kuromatsu: Pinus Thunbergii Parl.

43. Pilophorus niger Poppius, 1914

Jap. name: Ookuro-hyoutan-mekuragame

Spec. exam.: 1 ex., (A), L. T., 14. ix. 1992, K. Takeno leg.; 6 exs., (A), 12. x. 1992, K. Takeno leg.

Gen. distr.: Honshu, Kyushu and Tsushima Is.; Russia (Far East) and Mongolia.

Host.: [Kusunoki-ka: Lauraceae] Shirodamo: Neolitsea sericae (BI.) Koidz.

44. Pilophorus typicus (Distant, 1909)

Jap. name: Kuro-hyoutan-mekuragame

Spec. exam.: 1 ex., 18. vii. 1968, K. Kanmiya leg.; 1 ex., (0), sweeping, 8. xi. 1978, K. Takeno leg.

Gen. distr.: Honshu, Shikoku, Kyushu and Ryukyu Iss.; China, Hong Kong and S.E. Asia.

Host: Unknown. 
45. Pilophorus okamotoi Miyamoto et Lee, 1966***

Jap. name: Yomogi-hyoutan-mekuragame

Spec. exam.: Locality name only (through Miyamoto et Lee, 1966).

Gen. distr.: Kyushu and Tsushima Is.; Korea and Chejudo Is.

Host: [Kiku-ka: Compositae] Yomogi: Artemisia vulgalis L. var. indica Maxim.

\section{NABIDAE}

\section{Prostemmatinae}

46. Prostemma hilgendorffi Stein, 1878

Jap. name: Ashibuto-makiba-sashigame

Spec. exam.: 1 ex., 20. v. 1970, K. Takeno leg.; 1 ex., 22. v. 1970, K. Takeno leg.

Gen. distr.: Hokkaido, Honshu, Shikoku and Kyushu; Russia (Far East), Chejudo Is. and China.

Prey: Unknown.

\section{Nabis (Milu) apicalis (Matsumura, 1913)}

Jap. name: Kobane-makiba-sashigame

Spec. exam.: 1 ex., 13. vi. 1943, T. Esaki leg.; 3 exs., 29. v. 195 1, A. Habu leg.; 1 ex., 4th instar, 29. vii. 1963, S. Kimoto leg.; 4 exs., 7. ix. 1964, K. Takeno leg.: 1 ex., 8. ix. 1968, K. Kanmiya leg.; 2 exs., 16. vi. 1969, M. T. Chûjô leg.; 1 ex., 17. v. 1971, M. T. Chûjô leg.; 20. v. 1971, M. T. Chûjô leg.; 3 exs., (E), 11. v. 1972, K. Takeno leg.; 1 ex., (B-C), 25. vi. 1974, K. Takeno leg.; 1 ex., (M-O), 17. ix. 1974, K. Takeno leg.; 1 ex., (A-M), 4. iv. 1978, K. Takeno leg.; 1 ex., (D), 22. ix. 197, K. Takeno leg.; 1 ex., (C), 4. x. 1978, K. Takeno leg.; 1 ex., (A-V), 12. vi. 1979, K. Takeno leg.

Gen. distr.: Hokkaido, Honshu, Shikoku, Kyushu and Tsushima Is.

Prey: Unknown.

\section{Nabis stenoferus Hsiao, 1964}

Jap. name: Hanenaga-makiba-sashigame

Spec. exam.: 2 exs., 27. x. 1968, K. Kanmiya leg.; 1 ex., 12. xi. 1968, K. Kanmiya leg.; 1 ex., 18. xi. 1968, K. Kanmiya leg.; 4 exs., 12. iv. 1969, K. Kanmiya leg.: 1 ex., 20. vi. 1969, K. Kanmiya leg.; 1 ex., 11. vii. 1969, K. Kanmiya leg.; 1 ex., 25. vii. 1969, K. Kanmiya leg.; 1 ex., (A), light trap, 19. vi. 1975, K. Takeno leg.; 1 ex., (A), light trap, 4. vii. 1975, K. Takeno leg.

Gen. distr.: Hokkaido, Honshu, Shikoku and Kyushu; Russia (Far East).

Prey: Unknown. 


\section{ANTHOCORIDAE}

\section{Anthocorinae}

49. Anthocoris miyamotoi Hiura, 1959

Jap. name: Kimon-kuro-hanakamemushi

Spec. exam.: 1 ex., 20. viii. 1956, S. Miyamoto leg. (through Hiura, 1959).

Gen. distr.: Hokkaido, Honshu, Shikoku, Kyushu and Tokara Iss.; Russia (Far East) and Sakhalin.

Host: [Buna-ka: Fagaceae] Hahaso: Quercus serrata Thunb.

50. Elatophilus nipponensis Hiura, 1966

Jap. name: Hirata-hanakamemushi

Spec. exam.: 1 ex., 20. v. 1939, H. Hori leg. (through Hiura, 1966).

Gen. distr.: Honshu and Kyushu.

Prey: Unknown.

\section{Lyctocorinae}

5 1. Lasiochilus (Dilasia) japonicus Hiura, 1967

Jap. name: Kebuka-hanakamemushi

Spec. exam.: 1 ex., 21. xi. 1939, K. Yasumatsu leg. (through Hiura, 1967).

Gen. distr: Honshu, Shikoku, Kyushu, Tsushima Is. and Tokara Iss.

Prey: Unknown.

52. Amphiareus obscuriceps (Poppius, 1909)

Jap. name: Yasa-hanakamemushi

Spec. exam.: 3 exs., 15-16. viii. 1958, Y. Miyatake leg. (through Hiura, 1960).

Gen. distr.: Hokkaido, Honshu, Shikoku, Kyushu and Tsushima Is.; Kuril Iss., Russia (Far East), Korea (incl. Chejudo Is.).

Prey: Unknown.

\section{TINGIDAE}

\section{Tinginae}

53. Cochlochila conchata (Matsuura, 19 13)

Jap. name: Eguri-gunbai 
Spec. exam.: 46 exs., 20. v. 1939, K. Yasumatsu leg.; 14 exs., 2 copula, 19. v. 1943, T. Esaki, M. Tokunaga et S. Ito leg.; 1 ex., 30. v. 1943, K. Yasumatsu leg.; 2 exs., 25. v. 1965, K. Takeno leg.; 2 exs., (O-C), 25. v. 1974, K. Takeno leg.; 16 exs., 2 copula, (V), 2. v. 1978, K. Takeno leg.; 6 exs., (A), 5. vi. 1978, K. Takeno leg.

Gen. distr.: Hokkaido, Honshu and Kyushu; Russia (Far East), China and Korea.

Host: [Kiku-ka: Compositae] Otakarakou: Ligularia sibirica Cass., Akitabuki: Petusites japonicus subsp. gigunteus (Fr. Schum.) Kitam. (Ooi), Fuki: Petusites juponicum Miq.

\section{Copium japonicum Esaki, 1931}

Jap. name: Hige-buto-gunbai

Spec. exam.: 1 ex., 10. x. 1963, H. Kuroko leg.; 1 ex., 2 1. vii. 1968, K. Kanmiya leg.; 1 ex., 11. vii. 1969, K. Kanmiya leg.

Gen. distr.: Honshu, Shikoku and Kyushu; Formosa and S. China.

Host: [Kuchibirubana-ka: Labiatae] Inukoujyu: Mosla punctata Maxim., Shimobashira: Keisukeu juponicu Miq., Nigakusa: Teucrium juponicum Houtt.

\section{Cysteochila monstrosa (Scott, 1974)}

Jap. name: Koakaso-gunbai

Spec. exam.: 3 exs., 29. v. 195 1, A. Habu leg.; 2 exs., 20. vi. 1969, K. Kaniya leg.; 1 ex., 28. vii. 1969, K. Kanmiya leg.; 1 ex., 6. viii. 1969, K. Kanmiya leg.; 1 ex., 20. v. 1971, K. Takeno leg.; 8 exs., (G), 13. ix. 1972, K. Takeno leg.; 1 ex., (G), 22. ix. 1972, K. Takeno leg.; 5 exs., (B-C), 25. vi. 1974, K. Takeno leg.; 4 exs., (A-C-O), 26. vi. 1974, K. Takeno leg.; 1 ex., (A), 13. vii. 1974, K. Takeno leg.

Gen. distr.: Honshu, Shikoku, Kyushu and Tsushia Is.; China, Formosa and India.

Host: [Irakusa-ka: Urticaceae] Koakaso: Boehmeriu spicata Thunb., Yabumaou: Boehmeriu juponicu Miq.

56. Galeatus affinis (Herrich-Schaeffer, 1835)

Jap. name: Kiku-gunbai

Spec. exam.: 1 ex., 12. v. 1965, S. Kimoto leg.; 1 ex., 7. vii. 1968, K. Kanmiya leg.; 1 ex., 13. xi. 1968, K. Kanmiya leg.; 2 exs., 11. vii. 1969, K. Kanmiya leg.

Gen. distr.: Honshu, Shikoku, Kyushu and Tsushima Is.; Europe, Korea and Formosa.

Host: [Kiku-ka: Compositae] Yomogi: Artemisiu vulgaris L. var. indica Maxim., Kiku: Chrysanthemum morifolium Ramat, var. sinensis Makino, Arechinogiku: Erigeron linifolius Willd., Yomena-rui: Aster spp.

57. Stephanitis fasciicarina Takeya, 1963

Jap. name: Kusu-gunbai

Spec. exam.: 1 ex., 5. vii. 1939, K. Yasumatsu leg. 
Gen. distr.: Honshu, Shikoku, Kyushu, Tsushima Is., Goto Iss. and Okinawa Is.

Host: [Kusunoki-ka: Lauraceae] Gekkeijyu: Laurus nobilis Linnaeus, Shirodamo: Litsea glauca Sieb. et Zucc., Kusunoki: Cinnamomum cumphora Sieb., Yabunikkei: Cinnamomum japonicum Sieb.

58. Stephanitis hikosana Drake, 1948

Jap. name: Hikosan-gunbai

Spec. exam.: 3 exs., 21. vi. 1937, N. Takachiho leg.

Gen. distr.: Honshu, Kyushu and Yakushima Is.

Host: [Tsutsuji-ka: Ericaceae] Tsukushishakunage: Rhododendron Metternichii Sieb. et Zucc. (Ooi).

59. Stephanitis nasi Esaki et Takeya, 1931

Jap. name: Nashi-gunbai

Spec. exam.: 1 ex., 29. v. 1943, K. Yasumatsu leg.

Gen. distr.: Honshu, Shikoku and Kyushu; Russia (Far East), China and Korea.

Host: [Noibara-ka: Rosaceae] Anzu: Prunus armeniaca L. var. ansu Maxim., Rooo: Prunus Persicu Batsch., Someiyoshino: Prunus yedoensis Matsumura, Yamabuki: Kerria japonica DC., Sanzashi: Cretaegus cuneata Sieb, et Zucc., Boke: Chaenoeles lagenaria Koidz., Zumi: Malus Toringo Sieb., Kaidou: Malus micromalus Makino, Ringo: Malus pumila Mill var. dulcissima Koidz., Nashi: Pyrus Simonii Carr., [Roubai-ka: Calycanthaceae] Roubai: Meratica praecox Rehd. et Wils., [Kurumi-ka: Juglandaceae] Onigurumi: Juglans mandshrica Maxim. var. Sieboldiana Makino**.

60. Stephanitis pyrioides (Scott, 1974)

Jap. name: Tsutsuji-gunbai

Spec. exam.: 6 exs., 7. vii. 1939, K. Yasumatsu leg.

Gen. distr.: Honshu, Shikoku, Kyushu, Sado Is., Tsushima Is., Amakusa Is., Yakushima Is., Amami-Oshima Is. and Ryukyu Iss.; Russia (Far East), Korea, Formosa, S. China, Australia, Europe and N. America.

Host: [Kakinoki-ka: Ebenaceae] Kaki: Diospyros Kuki L. fil., [Tsutsuji-ka: Ericaceae] Nejiki: Pieris elliptica Nakai**, Tsutsuji-rui: Rhododendron spp.

61. Stephanitis svensoni Drake, 1948

Jap. name: Shikimi-gunbai

Spec. exam.: 27 exs., 17. ix. 1948, S. Miyamoto leg.; 2 exs., 16. vi. 1964, S. Kimoto leg.; 1 ex., 20. vi. 1969, K. Kanmiya leg.; 2 exs., (D), 21. vii. 1978, K. Takeno leg.; 1 ex., (A), 24. i. 1979, K. Takeno leg.

Gen. distr.: Honshu, Shikoku, Kyushu and Yakushima Is.

Host: [Mokuren-ka: Magnoliaceae] Shikimi: Zllicium religiosum Sieb. et Zucc. 
62. Stephanitis takeyai Drake et Maa, 1953

Jap. name: Tosaka-gunbai

Spec. exam.: 1 ex., 8. viii. 1938, K. Yasumatsu leg.; 4 exs., 24. ix. 1938, K. Yasumatsu leg.; 1 ex., 2. vi. 1939, K. Yasumatsu leg.; 2 exs., 30. vii. 1939, K. Yasumatsu leg.; 14 exs., 10. vi. 1939, K. Yasumatsu leg.; 2 exs., 30. vii. 1939, K. Yasumatsu leg.; 14 exs., 10. vi. 1945, K. Yasumatsu leg.; 5 exs., 16. vi. 1964, S. Kimoto leg.; 9 exs., 18. vi. 1965, K. Takeno leg.; 10 exs., 23. vi, 1965, K. Takeno leg.; 12 exs., I. ix. 1966, K. Takeno leg.; 9 exs., 13. ix. 1966, K. Takeno leg.; 3 exs., 18. vii. 1967, K. Takeno leg.; 13 exs., 6. vi. 1968, K. Takeno leg.; 2 exs., 17. vii. 1968, K. Takeno leg.; 16 exs., (G), 22. ix. 1972, K. Takeno leg.; 5 exs., (A), 10. vi. 1974, K. Takeno leg.; 1 ex., (A), 26. v. 1972, K. Takeno leg.; 21 exs., (A), 5. vi. 1978, K. Takeno leg.; 3 exs., (A), 10. vii. 1978, K. Takeno leg., 6 exs., (D), 2 1. vii. 1978, K. Takeno leg.

Gen. distr.: Honshu, Shikoku, Kyushu, Yakushima Is. and Amami-Oshima Is.; America.

Host: [Egonoki-ka: Styracaceae] Egonoki: Styrax juponica Sieb. et Zucc., [Kakainokika: Ebenaceae] Kaki: Diospyros Kuki Linnaeus fil., [Tsutsuji-ka: Ericaceae] Asebi: Pieris juponica D. Don, Nejiki: Pieris elliptica Nakai, Hotsutsuji: Tripetuleia paniculata Sieb. et Zucc., [Tochinoki-ka: Hippocastanaceae] Tochinoki: Aesculus turbinutu Blume, [Ibaraka: Rosaceae] Yamazakura: Prunus donarium Sieb. var. spontanea Makino, Ushikoroshi: Pourthiueu villosu Decne., [Yukinoshita-ka: Saxifragaceae] Iwagarami: Schizophrugmu hydrungeoides Sieb. et Zucc., [Kusunoki-ka: Lauraceae] Shiromoji: Purubenzoin trilobum Nakai, Aburachan: Parabenzoin pruecox Nakai, Kuromoji: Benzoin umbellatum Rehd., Ukonbana: Benzoin obtusilobum 0. Kuntze., Kusunoki: Cinnamomum Camphora Sieb., Kekuromoji: Linderu sericea (Sieb. et Zucc.) Blume. (Ooi), Aomoji: Linderu citriodoru (Sieb. et Zucc.) Hemsl. (Ooi), Kanakuginoki: Benzoin erythrocurpus Rehd., [Mokuren-ka: Magnoliaceae] Shikimi: Lillicium religiosum Sieb. et. Zucc.

63. Tingis (Tingis) ampliata (Herrich-Schaeffer, 1839) Jap. name: Azami-gunbai

Spec. exam.: 1 ex., (at 1000m in altitude), 19. iv. 1938, K. Yasumatsu leg.; 11 exs., 2. vi. 1939, K. Yasuatsu leg., 14 exs., 5. vi. 1939, K. Yasumatsu leg.; 1 ex., 2. viii. 1957, H. Kuroko leg.; 1 ex., 12. v. 1965, K. Takeno leg.; 1 ex., 25. v. 1965, K. Takeno leg.; 1 ex., 16. vi. 1967, K. Takeno leg.; 1 ex., (G), 22. ix. 1972, K. Takeno leg.; 1 ex., 25. v. 1974, K. Takeno leg.

Gen. distr.: Honshu, Shikoku and Kyushu; Formosa and Palaearctic Region.

Host: [Kiku-ka: Compositae] Azami-rui: Circium spp., Hireazami-mi: Cardus spp. 
64. Tingis (Tingis) crispata (Herrich-Schaeffer, 1838)

Jap. name: Yomogi-gunbai

Spec. exam.: 3 exs., 23. viii. 1961, S. Kimoto leg.; 1 ex., 25. iv. 1965, M. Shiga leg.

Gen. distr.: Honshu, Shikoku and Kyushu; China, Korea Formosa and Palaearctic Region.

Host: [Kiku-ka: Compositae] Yomogi: Artemisia vulgaris L. var. indica Maxim.

65. Uhlerites debilis (Uhler, 1896)

Jap. name: Hime-gunbai

Spec. exam.: Locality name only (through Takeya, 1962).

Gen. distr.: Hokkaido, Honshu, Shikoku and Kyushu; Russia (Far East), China, Korea and Formosa.

Host: [Buna-ka: Fagaceae] Hahaso: Quercus serruta Thunb., Ubamegashi: Quercus phyllirueoides A. Gray, Kunugi: Quercus ucutissima Carruth., Kashiwa: Quercus dentata Thunb., Kuri: Castanea pubinervis Schneid.

\section{REDUVIIDAE}

\section{Harpactorinae}

66. Zsyndus obscurus (Dallas, 1850)

Jap. name: Ootobi-sashigame

Spec. exam.: 2 exs., 6. xi. 1938, K. Yasumatsu leg.; 1 ex., 30. x. 1956, H. Kuroko leg.; 1 ex., 22. viii. 1963, S. Kimoto leg.; 1 nymph, 1. ix. 1964, K. Takeno leg.; 1 ex., 27. x. 1964, K. Takeno leg.; 1 ex., 28. x. 1964, K. Takeno leg.; 1 ex., 6. iv. 1967, K. Takeno leg.; 1 ex., 6. iv. 1967, T. Teshima leg.; 1 ex., 17. ix. 1978, T. Teshima leg.; 1 ex., 10. v. 1970, M. T. Chûjô leg.; 1 ex., 19. iii. 197 1, K. Takeno leg.; 1 ex., 25. x. 197 1, K. Takeno leg.; 1 ex., (A), 2. xi. 197 1, K. Takeno leg.; 1 ex., 3. v. 1972, K. Takeno leg.; 1 ex., (M-P), 17. x. 1974, K. Takeno leg.; 1 ex., (C-O), 7. xi. 1974, K. Takeno leg.; 1 ex., (A), 10. iv. 1975, K. Takeno leg.; 1 ex., (A), 17. iii. $1978 \mathrm{~K}$. Takeno leg.; 1 ex., (A), 21. iv. 1979, K. Takeno leg.; 1 ex., (A), 9. iv. 1980, K. Takeno leg.

Gen. distr.: Honshu, Shikoku and Kyushu; China, Korea, Formosa and Bhutan.

Prey: Miscellaneous small insects.

\section{Cydnocoris russatus Stal, 1866}

Jap. name: Aka-sashigame

Spec. exam.: 1 ex., 20. ix. 1937, K. Yasumatsu leg.; 1 ex., 24. ix. 1938, K. Yasumatsu leg.; 1 ex., 19. v. 1943, T. Esaki et S. Ito leg.; 1 ex., 18. vi. 195 1, A. Habu 
leg.; 1 ex., 18. v. 1954, H. Kuroko leg.; 1 ex., 15. ix. 1964, K. Takeno leg.; 1 ex., 24. ix. 1964, K. Takeno leg.; 1 ex., 28. ix. 1964, K. Takeno leg.; 1 ex., 26. x. 1964, K. Takeno leg.; 1 ex., 11. xi. 1964, K. Takeno leg.; 1 ex., 21. v. 1965, K. Takeno leg.; 1 ex., 17. v. 1967, K. Takeno leg.; 1 ex., 17. vii. 1968, K. Takeno leg.; 1 ex., 14. v. 1969, K. Takeno leg.; 1 ex., 3. v. 1971, M. T. Chujo leg.; 1 ex., 22. x. 1971, K. Takeno leg.; 2 exs., (A), 19. vi. 1974, K. Takeno leg.; 1 ex., (A), 1. vii. 1975, K. Takeno leg.; 1 ex., 10. v. 1976, K. Takeno leg.; 1 ex., (V), 7. vii. 1976, K. Takeno leg.; 1 ex., (A), 8. vii. 1978, K. Takeno leg.; 1 ex., (A), 2. x. 1979, K. Takeno leg.; 1 ex., (A), sweeping, 2. xi. 1979, K. Takeno leg.; 1 ex., (A), 15. v. 1985, K. Takeno leg.

Gen. distr.: Honshu, Shikoku, Kyushu and Tsushima Is.; China and Formosa.

Prey: Larvae of Lepidoptera Gen. et spp., Tortricidae Gen, et spp. and Coleoptera Gen. et spp.

\section{Sphedanolestes impressicollis (Stal, 1861)}

Jap. name: Shima-sashigame

Spec. exam.: 1 ex., (at 650m in altitude), 10. v. 1939, K. Yasumatsu leg.; 1 ex., 25. vi. 1951, A. Habu leg.; 1 ex., 25. vi. 1965, K. Takeno leg.; 1 ex., 13. vii. 1966, T. Teshima leg.; 1 ex., 25. vi. 1967, T. Teshima leg.; 2 exs., (I), 6. vi. 1972, K. Takeno leg.; 1 ex., (N), 14. vi. 1972, K. Takeno leg.; 3 exs., (A), 7. vi. 1975, K. Takeno leg.; 4 exs., (A), 19. vi. 1975, K. Takeno leg.; 1 ex., (A), 20. vi. 1975, K. Takeno leg.; 4 exs., (A), 19. vi. 1975, K. Takeno leg.; 1 ex., (A), 20. vi. 1975, K. Takeno leg.; 1 ex., (A), 13. vii. 1975, K. Takeno leg.; 1 ex., (A-D), 6. viii. 1975, K. Takeno leg.; 1 ex., (D), 6. viii. 1976, K. Takeno leg.; 1 ex., (A), 16. vi. 1979, K. Takeno leg.; 3 exs., (AV), 12. vi. 1979, K. Takeno leg.; 1 coupla, 13. vi. 1979, K. Takeno leg.

Gen. distr.: Honshu, Shikoku, Kyushu and Tsushima Is.; China.

Prey: [Hamushi-ka: Chrysomelidae] Ruri-hamushi: Linaeidea aenea (Linnaeus), Yomogi-hamushi: Chrysolinu artichalcea (Mannerheim), Aobane-sat-u-hamushi: Basilepta fulvipes (Motschulsky), Higenaga-rurimaru-nomi-hamushi: Hemipyxis plugioderoides (Motschulsky), Azami-kaminari-hamushi: Altica cirsicolu Ohno, [Tentoumushi-ka: Coccinellidae] Nanahoshi-tentou: Coccinellu septempunctutu Linnaeus, miscellaneous Coleoptera Gen. et spp., and miscellaneous larvae of Lepidoptera; Host: [Karakasabanaka: Umbelliferae] Ninjin: Daucus Curotu L. var. sutivu DC.

\section{Velinus nodipes (Uhler, 1860)}

Jap. name: Yani-sashigame

Spec. exam.: 2 exs., 5. vi. 1939, K. Yasumatsu leg.; 2 exs., (D-E), 7. vi. 1974, K. Takeno leg.; 1 ex., (A), 20. vi. 1974, K. Takeno leg.

Gen. distr.: Honshu, Shikoku and Kyushu; China and Korea.

Prey: [Hamushi-ka: Chrysomelidae] Ruri-hamushi: Linaeidae aenea (Linnaeus), and miscellaneous small insects. 


\section{R eduviinae}

\section{Reduvius humeralis (Scott, 1874)}

Jap. name: Kubi-aka-sashigame

Spec. exam.: 2 exs., 5. vii. 1939, K. Yasumatsu leg.; 2 exs., 6. vii. 1942, Esaki, Masaki et Ishihara leg.; 1 ex., 11. vi. 1955, H. Kuroko leg.; 1 ex., light trap, 21. vii. 1969, M. T. Chûjô leg.; 1 ex., 29. vii. 1970, T. Teshima leg.; 1 ex., 4. viii. 1970, K. Takeno leg.; 1 ex., 25. vi. 1974, K. Takeno leg.; 1 ex., 26. vi. 1974, K. Takeno leg.; 1 ex., 29. vi. 1974, K. Takeno leg.; 1 ex., (A), light trap, 3. vii. 1975, K. Takeno leg.; 1 ex., (A), light trap, 17. vii. 1975, K. Takeno leg.; 1 ex., 20. vii. 1975, K. Takeno leg.; 1 ex., 28. vi. 1976, K. Takeno leg.; 1 ex., (A), light trap, 2. viii. 1977, K. Takeno leg.; 1 ex., (A), light trap, 12. vii. 1979, K. Takeno leg.

Gen. distr.: Honshu, Shikoku, Kyushu and Ryukyu Iss.

Prey: Miscellaneous larvae of Lepidoptera Gen. et spp.

\section{Stenopodinae}

\section{1. Oncocephalus assiilis Reuter, 1882}

Jap. name: Tobiiro-sashigame

Spec. exam.: 1 ex., 1. vii. 195 1, A. Habu leg.; 1 ex., 3. ix. 1964, S. Kimoto leg.; 2 exs., 5. ix. 1964, S. Kimoto leg.; 1 ex., 21. vii. 1968, K. Kanmiya leg.; 1 ex., (A), light trap, 21. vii. 1969, M. T. Chujo leg.; 1 ex., (A), light trap, 2 1. vii. 1974, K. Ohara leg.; 1 ex., (A), 3. vii. 1975, K. Takeno leg; 2 exs., (A), light trap, 13. ix. 1975, K. Takeno leg.; 2 exs., (A), 31. iii. 1979, K. Takeno leg.; 1 ex., (A), 2. v. 1979, K. Takeno leg.

Gen. distr.: Honshu, Shikoku and Kyushu; Philippines.

Prey: Unknown.

\section{Pygolampis bidentata (Goeze, 1778)}

\section{Jap. name: Hoso-sashigame}

Spec. exam.: 1 ex., 12. vii. 1939, K. Yasumatsu leg.; 1 ex., 1. vii. 195 1, A. Habu leg.; 1 ex., 28. vii. 195 1, A. Habu leg.; 1 ex., 29. vii. 1956, H. Kuroko leg.; 1 ex., 25. vii. 1964, S. Kimoto leg.; 1 ex., 9. vi. 1970, T. Teshima leg.; 1 ex., (A), 13. vi. 1975, K. Takeno leg.; 2 exs., (A), light trap, 4. vii. 1975, K. Takeno leg.; 1 ex., (A), 20. vii. 1978, K. Takeno leg.

Gen. distr.: Hokkaido, Honshu, Shikoku, Kyushu and Ryukyu Iss.; E. Siberia and Formosa.

Prey: Unknown. 


\section{Emesinae}

\section{Myiophanes tipulina Reuter, 1881}

Jap. name: Gomi-ashi-naga-sashigame

Spec. exam.: 1 ex., 26. xi. 1949, A. Habu leg.; 1 ex., 1. viii. 195 1, A. Habu leg.: 1 ex., 18. vi. 1955, H. Kuroko leg.; 1 ex., 30. ix. 1958, H. Kuroko leg.; 1 ex., (A), 26. viii. 1974, K. Ohara leg.

Gen. distr.: Honshu, Shikoku and Kyushu; China, Korea and Australia.

Prey: Unknown.

\section{Schidium marcidum (Uhler, 1896)***}

Jap. name: Ashinaga-sashigame

Spec. exam.: 1 ex., 8. vii. 1939, K. Yasumatsu leg.; 1 ex., 10. vii. 1956, H. Kuroko leg.; 1 ex., beating, 27. i. 1978, K. Takeno leg.

Gen. distr.: Honshu, Shikoku, Kyushu and Tsushima Is.; China, Korea, S.-E. Asia and Australia.

Host?: Filices spp.

\section{Ectrychodiinae}

75. Ectrychotes andreae (Thunberg, 1784)

Jap. name: Biroudo-sashigame

Spec. exam.: 1 ex., 3 1. vi. 1971, K. Takeno leg.

Gen. distr.: Honshu, Shikoku, Kyushu, Tsushima Is. and Okinawa Is.; China and Korea.

Prey: Unknown.

76. Haematoloecha delibuta (Distant, 1883)

Jap. name: Kubi-guro-aka-sashigame

Spec. exam.: 1 ex., 24. v. 1937, K. Yasumatsu leg.: 1 ex., 17. vii. 195 1, A. Habu leg.; 1 ex., 5. v. 1965, Y. Kajita leg.; 1 ex., 13. v. 1965, K. Takeno leg.; 1 ex., 16. v. 1967, T. Teshima leg.; I ex., 14. v. 1968, K. Takeno leg.; 1 ex., 12. iii. 1971, M. T. Chujo leg.; 1 ex., 11. v. 1972, K. Takeno leg.; 4 exs., (A), 28. iv. 1976, K. Takeno leg.; 1 ex., (A), 10. v. 1976, K. Takeno leg.; 1 ex., 30. iv. 1979, K. Takeno leg.

Gen. distr.: Honshu, Shikoku and Kyushu.

Host: [Azami-rui: Musci spp.]

77. Haematoloecha nigrorufa (Stal, 1866)

Jap. name: Akashima-sashigame

Spec. exam.: 1 ex., (D-B), 18. ix. 1974, K. Takeno leg. 
Gen. distr.: Honshu, Shikoku, Kyushu, Tsushima Is., Ishigaki Is. and Yonaguni Is.; China and Korea.

Prey: Baikyaku-rui: Diplopoda spp.

78. Labidocoris insignis Distant, 1883

Jap. name: Kuro-ba-aka-sashigame

Spec. exam.: 1 ex., 13. vi. 1954, H. Kuroko leg.; 1 ex., 27. v. 1970, K. Takeno leg.

Gen. distr.: Honshu, Shikoku and Kyushu.

Prey: Unknown.

\section{Labidocoris pectoralis (Stal, 1863)}

Jap. name: Ashi-aka-kuroba-sashigame

Spec. exam.: 1 ex., 21. vii. 1950, A. Habu leg.

Gen. distr.: Honshu and Kyushu; China.

Prey: Unknown.

\section{Peiratinae}

80. Peirates turpis Walker, 1873

Jap. name: Kuromonsashigame

Spec. exam.: 1 ex., 18. vii. 1939, K. Yasumatsu leg., 1 ex., 20. vii. 1963, S. Kimoto leg., 1 ex., 29. vii. 1963, S. Kimoto leg.; 1 nymph, 14. ix. 1967, K. Takeno leg.; 1 ex., 20. v. 1968, K. Takeno leg.; 1 nymph, 10. vi. 1969, M. T. Chujo leg.; 1 nymph, 16. v. 1970, T. Teshima leg.; 1 ex., 26. v. 1970, K. Takeno leg.; 1 ex., 2. viii. 1970, M. T. Chujo leg.; 3 exs., 1. vi. 1972, K. Takeno leg.; 1 ex., (N), 5. vi. 1972, K. Takeno leg.; 1 ex., (A), 1.v. 1975, K. Takeno leg.; 1 ex., 5. vi. 1978, K. Takeno leg.; 1 ex., 10. vi. 1980, K. Takeno leg.

Gen. distr.: Honshu, Shikoku, Kyushu, Tsushima Is. and Ryukyu Iss.; China and Korea.

Prey: [Hishibatta-ka: Tetrigidae Gen. et spp.], [Gomimushi-ka: Harpalidae Gen. et spp.].

\section{ARADIDAE}

\section{Aradinae}

81. Aradus orientalis Bergroth, 1885

Jap. name: Nokogiri-hirata-kamemushi

Spec. exam.: 11 exs., (at 1000m in altitude), 19. iv. 1938, K. Yasumatsu leg.; 3 exs., (at $900 \mathrm{~m}$ in altitude), 26. iv. 1938, K. Yasumatsu leg.; 9 exs., 27. v. 1938, Esaki, 
Nomura et Yasumatsu leg.; 6 exs., 31. v. 1938, Esaki, Nomura et Yasumatsu leg.; 1 ex., 9. vii. 1938, K. Yasumatsu leg.; 1 ex., 16. v. 1957, H. Kuroko leg.; 1 ex., 26. iv. 1958, H. Kuroko leg.; 1 ex., 1. vi. 1958, H. Kuroko leg.; 1 ex., 25. iv. 1965, A. Taketani leg.; 1 ex., 25. iv. 1965, S. Kimoto leg.; 1 ex., 6. viii. 1969, K. Kanmiya leg.; 1 ex., 13. x. 1969, M. T. Chûjô leg.; 2 exs., 25. viii. 1970, M. T. Chûjô leg.; 1 ex., 11. v. 1971, M. T. Chûjô leg.; 1 ex., 17. v. 1971, M. T. Chûjô leg.; 1 ex., 12. iv. 1972, K. Takeno leg.; 1 ex., 24. iv. 1972, K. Takeno leg.; 1 ex., (D), 28. iv. 1972, K. Takeno leg.; 10 exs., 11. v. 1972, K. Takeno leg.; 1 ex., (A), 20. vi. 1974, K. Takeno leg.; 2 exs., 25. vi. 1974, K. Takeno leg.; 1 ex., (A), 23. viii. 1974, K. Takeno leg.

Gen. distr.: Hokkaido, Honshu, Shikoku and Kyushu.

Host: [Hiyu-ka Amaranthaceae] Sennichikou: Gomphrena globosa Linnaeus, [Sarunokoshikake-ka: Polyporaceae] Masutake: Laetiporus sulphureus (Fr..) Bond. et Sing. var. miniatus (Junghu.) Imaz., Kawaratake: Corilus versicolor (Fr.) Quel., Shutake: Polustictus cinnabarinus (Jacq.) Fr., Kaigaratake: Lenzites betulia Fr., [Shimejika: Tricholomataceae] Hiratake: Pleurotus ostreatus (Fr.) Quel**, Shiitake: Lentinus edodes (Berk.) Sing., Matsutake: Tricholoma matsutuke (S. Ito et Imai) Sing.

\section{Aradus unicolor Kiritshenko, 1913}

Jap. name: Matsu-hirata-kamemushi

Spec. exam.: 4 exs., 3. vi. 1939, K. Yasumatsu leg.

Gen. distr.: Honshu, Kyushu and Tsushima Is.; E. Siberia.

Host: [Sarunokoshikake-ka: Polyporaceae] Hitokuchitake: Cryptoporus volvatus (Pk.).

\section{Mezirinae}

\section{Mezira taiwanica Kormilev, 1957}

Jap. name: Kuro-hirata-kamemushi

Spec. exam.: 6 exs., 4. vii. 1969, M. T. Chujo leg.; 1 ex., 3. vii. 1970, K. Takeno leg.

Gen. distr.: Hokkaido, Honshu, Shikoku, Kyushu, Tsushima Is. and Bonin Iss.; China, Korea, Formosa, Micronesia and India.

Host: Unknown. 